\title{
Incidence and implications of postoperative supraventricular tachycardia after pulmonary lobectomy
}

\author{
Gregory P. Giambrone, MS, ${ }^{\mathrm{a}}$ Xian Wu, MPH, ${ }^{\mathrm{b}}$ Licia K. Gaber-Baylis, BA, ${ }^{\mathrm{a}}$ Akshay U. Bhat, MEng, \\ Ramin Zabih, PhD, ${ }^{\mathrm{c}}$ Nasser K. Altorki, MD, ${ }^{\mathrm{d}}$ Peter M. Fleischut, MD, and Brendon M. Stiles, MD
}

\begin{abstract}
Objective: We sought to determine the rate of postoperative supraventricular tachycardia (POSVT) in patients undergoing pulmonary lobectomy, and its association with adverse outcomes.

Methods: Using the State Inpatient Database, from the Healthcare Cost and Utilization Project, we reviewed lobectomies performed (2009-2011) in California, Florida, and New York, to determine POSVT incidence. Patients were grouped by presence or absence of POSVT, with or without other complications. Stroke rates were analyzed independently from other complications. Multivariable regression analysis was used to determine factors associated with POSVT.
\end{abstract}

Results: Among 20,695 lobectomies performed, 2449 (11.8\%) patients had POSVT, including 1116 (5.4\%) with isolated POSVT and 1333 (6.4\%) with POSVT with other complications. Clinical predictors of POSVT included age $\geq 75$ years, male gender, white race, chronic obstructive pulmonary disease, congestive heart failure, thoracotomy surgical approach, and pulmonary complications. POSVT was associated with an increase of: stroke (odds ratio [OR] 1.74; 95\% confidence interval [CI] 1.03-2.94); in-hospital death (OR 1.85; 95\% CI 1.45-2.35); LOS (OR 1.33; 95\% CI 1.29-1.37); and readmission (OR 1.29; $95 \%$ CI 1.04-1.60). The stroke rate was $<1 \%$ in patients who had isolated POSVT, and $1.5 \%$ in patients with POSVT with other complications. Patients with isolated POSVT had increased readmission and LOS, and a marginal increase in stroke rate, compared with patients with an uncomplicated course.

Conclusions: POSVT is common in patients undergoing pulmonary lobectomy and is associated with adverse outcomes. Comparative studies are needed to determine whether strict adherence to recently published guidelines will decrease the rate of stroke, readmission, and death after POSVT in thoracic surgical patients. (J Thorac Cardiovasc Surg 2016;151:982-9)

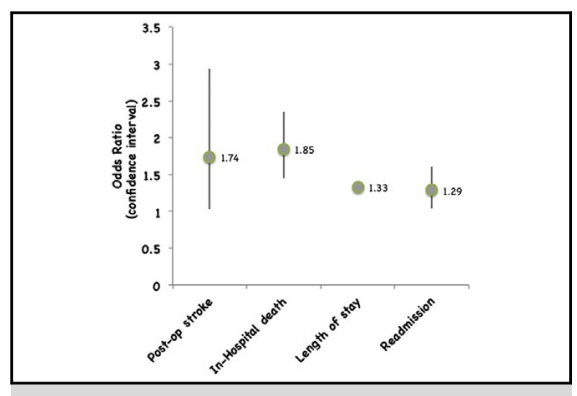

Postoperative supraventricular tachycardia is associated with adverse outcomes after pulmonary lobectomy.

\section{Central Message}

Postoperative supraventricular tachycardia is associated with adverse outcomes after pulmonary lobectomy.

\section{Perspective}

Postoperative atrial arrhythmias are common after pulmonary lobectomy, and they are associated with stroke, death, increased length of stay, and readmission. Identifying at-risk patients will allow for tailored prophylaxis strategies.

See Editorial Commentary page 990.

See Editorial page 913.
New-onset supraventricular tachycardias (SVTs), including atrial fibrillation and atrial flutter, are common in patients after pulmonary lobectomy. ${ }^{1-3}$ Postoperative SVT (POSVT) carries several nonmodifiable risk factors, including advanced age, male gender, white race, and history of arrhythmias. $^{1-5}$ Modifiable preoperative risk factors

\footnotetext{
From the ${ }^{\mathrm{a}}$ Department of Anesthesiology, NewYork-Presbyterian Hospital, Weill Cornell Medicine, New York; ${ }^{b}$ Department of Healthcare Policy and Research, Division of Biostatistics and Epidemiology, Weill Cornell Medicine, New York; ${ }^{\mathrm{c}}$ Department of Computer Science, Cornell University, Ithaca; and ${ }^{\mathrm{d}}$ Department of Cardiothoracic Surgery, Thoracic Division, NewYork-Presbyterian Hospital, Weill Cornell Medicine, New York, NY.

N.K.A., P.M.F., and B.M.S. are co-senior authors.

Funding for this work was provided by the Center for Perioperative Outcomes, Department of Anesthesiology at Weill Cornell Medicine, NewYorkPresbyterian Hospital. Xian Wu, MPH, was partially supported by the following grant: Clinical and Translational Science Center at Weill Cornell Medicine (UL1-TR000457-06).
}

include medical control of hypertension and congestive heart failure, tobacco and alcohol use, and the surgical approach used. Modifiable postoperative risk factors include fluid overload, hypoxia, and heart rate control.

Postoperative SVT is associated with increased morbidity, length of stay (LOS), and cost, in patients undergoing

\footnotetext{
Read at the 95th Annual Meeting of The American Association for Thoracic Surgery, Seattle, Washington, April 25-29, 2015.

Received for publication May 5, 2015; revisions received Oct 2, 2015; accepted for publication Nov 21, 2015; available ahead of print Jan 14, 2016.

Address for reprints: Brendon M. Stiles, MD, Department of Cardiothoracic Surgery,

Division of Thoracic Surgery, Weill Cornell Medicine, NewYork-Presbyterian

Hospital, Ste M404, 525 East 68th St, New York, NY 10021 (E-mail: brs9035@ med.cornell.edu).

$0022-5223 / \$ 36.00$

Copyright (C) 2016 by The American Association for Thoracic Surgery

http://dx.doi.org/10.1016/j.jtcvs.2015.11.057
} 


\section{Abbreviations and Acronyms \\ AATS = American Association for Thoracic Surgery \\ CI $=$ confidence interval \\ ICD-9 = International Classification of \\ Diseases, ninth edition \\ LOS = length of stay \\ OR $=$ odds ratio \\ POSVT $=$ postoperative SVT \\ SID-HCUP $=$ State Inpatient Database, Healthcare Cost and Utilization Project \\ STS $=$ Society of Thoracic Surgeons \\ SVT = supraventricular tachycardia}

Scanning this QR code will take you to the article title page. To view the AATS 2015 Webcast, see the URL at the end of the article.

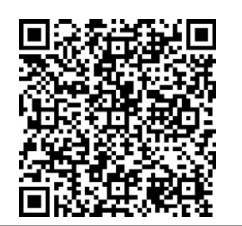

thoracic surgery. ${ }^{1,3,6}$ Because POSVT represents a potentially preventable adverse outcome, the American Association for Thoracic Surgery (AATS) ${ }^{7}$ and the Society of Thoracic Surgeons (STS) ${ }^{8}$ recently published evidencebased guidelines for the prophylaxis and treatment of POSVT in patients undergoing general thoracic surgical procedures. In the AATS guidelines, ${ }^{7}$ both open and thoracoscopic lobectomy were classified as high-risk procedures for POSVT, with an estimated incidence $>15 \%$. Thus, the guidelines recommended that these patients be monitored continuously for $\geq 48$ to 72 hours, and that pharmacologic prophylaxis be considered. However, individual risk likely spans a spectrum from low to high risk.

To perform a broad investigation of POSVT after pulmonary lobectomy, we utilized the State Inpatient Databases from the Healthcare Cost and Utilization Project (SIDHCUP). ${ }^{9}$ We anticipate that a better understanding of factors associated with POSVT in this large patient population will allow for better risk stratification of patients, for both POSVT and stroke after POSVT. For example, the influence of patient-based, versus perioperative, factors on the risk of POSVT is unknown. Additionally, the risk of isolated POSVT is largely unknown.

We therefore sought to compare the clinical outcomes of isolated POSVT with those of POSVT that is associated with other postoperative complications. Isolated POSVT has been suggested to have little independent association with adverse postoperative outcomes. ${ }^{3}$ The objective of the current study is to generate hypotheses for clinical comparative studies, to evaluate whether adherence to AATS guidelines influences the rate of POSVT and associated complications.

\section{METHODS \\ Database and Study Population}

We examined hospitalizations for adults (aged $\geq 18$ years), using SIDHCUP discharge data from the period 2009 to 2011, from California, Florida, and New York. All study activities were approved by the Weill Cornell Medicine Institutional Review Board. The SID-HCUP is an all-payer inpatient database, containing discharges from nonfederal, nonpsychiatric community hospitals. This database has been described previously, but briefly the SID contains $>100$ clinical and nonclinical variables, such as principal and secondary diagnoses and procedures, admission and discharge status, patient demographics, LOS, and total charges. ${ }^{9,10}$

To identify the difference between pre-existing diagnoses and complications occurring after hospital admission, a unique identifier corresponding to each diagnosis code was used. For analysis of uncomplicated versus complicated POSVT, the patient cohort was divided into 4 groups: (1) no POSVT or other complications; (2) only POSVT as a complication; (3) other complications but no POSVT; and (4) other complications and POSVT. For the purposes of segregating patients into the 4 groups, we excluded stroke as a complication, to determine any association of isolated POSVT with stroke. To determine variability in rates of POSVT in the patient cohort, based on surgical volume of the hospitals at which the lobectomy was performed, hospitals were divided into quartiles and analyzed. The quartiles were based on number of cumulative cases performed during the 3 -year period.

\section{Clinical Modification Codes}

Codes from the International Classification of Diseases, ninth edition (ICD-9), were used to identify patients undergoing pulmonary lobectomy: open lobectomy (or other lobectomy of lung [32.49]; lobectomy of lung [32.4] while excluding reopening of thoracotomy [34.03]; thoracoscopic lobectomy [32.41]; transpleural thoracoscopy [34.21]; and robot-assisted procedure $[17.4 \mathrm{x}]$ ) or minimally invasive lobectomy (MIL) (thoracoscopic lobectomy [32.41 while excluding $32.4,32.49,34.03$, and $17.4 \mathrm{x}]$ and robotic lobectomy [17.4x and 32.4,32.41, or 32.49 while excluding 34.03 and 34.21]). Similarly, ICD-9M codes were used to identify patients with POSVT not present on admission: atrial fibrillation and flutter (427.3); atrial fibrillation (427.31); atrial flutter (427.32); and paroxysmal SVT (427.0). Each diagnosis was coded with a separate identifier denoting whether it was present on admission, allowing for discrimination between pre-existing diagnoses and those occurring after admission. Records indicating SVT upon admission were excluded from further analyses. Additionally, patients aged $\leq 17$ years, and those with missing age or gender data, were excluded.

\section{Covariates}

Data were obtained for patient demographics (age, gender, race, state, and insurance status), LOS, comorbidities (with the indication of being present on admission), and in-hospital events that were not present on admission. The latter category includes supraventricular arrhythmia, myocardial infarction, deep venous thrombosis, pulmonary embolism, pneumonia, pulmonary collapse, empyema with or without fistula, mechanical ventilation, noninvasive ventilation, tracheostomy, sepsis/shock, urinary tract infection, accidental puncture or laceration complicating surgery, and bleeding complicating surgery. Postoperative in-hospital events included: stroke, acute respiratory insufficiency, acute pneumothorax, pulmonary edema, and wound infection. Baseline comorbidities were compared, using the modified Deyo index, which adapts, for administrative data, the Elixhauser Comorbidity Index, the commonly used Charlson Comorbidity Index for predicting the adjusted relative risk of 1-year mortality. ${ }^{11,12}$

\section{Statistical Analysis}

We analyzed patient baseline, demographic, clinical, and postoperative characteristics, using frequencies and proportions for categoric variables, 
TABLE 1. Characteristics of patients undergoing pulmonary lobectomy with or without postoperative supraventricular tachycardia

\begin{tabular}{|c|c|c|c|}
\hline Variable & No POSVT $(n=18,246)$ & POSVT $(n=2449)$ & $P$ value \\
\hline Age, median (Q1; Q3) & $67(59 ; 74)$ & $72(66 ; 78)$ & $<.001$ \\
\hline Male gender & $8027(44.0)$ & $1333(54.4)$ & $<.001$ \\
\hline White race & $13,850(75.9)$ & $2071(84.6)$ & $<.001$ \\
\hline Mean Charlson/Deyo index $(95 \% \mathrm{CI})$ & $3.43(3.40 ; 3.47)$ & $3.72(3.62 ; 3.83)$ & $<.001$ \\
\hline Median Charlson/Deyo index (Q1; Q3) & $3(2 ; 3)$ & $3(2 ; 4)$ & $<.001$ \\
\hline Chronic obstructive pulmonary disease & $7712(42.3)$ & $1253(51.2)$ & $<.001$ \\
\hline Renal failure & $863(4.7)$ & $184(7.5)$ & $<.001$ \\
\hline Congestive heart failure & $381(2.1)$ & $98(4.0)$ & $<.001$ \\
\hline Valvular heart disease & $532(2.9)$ & $167(6.8)$ & $<.001$ \\
\hline Pulmonary circulation disorders & $139(0.8)$ & $65(2.7)$ & $<.001$ \\
\hline Peripheral vascular disorders & $1161(6.4)$ & $223(9.1)$ & $<.001$ \\
\hline Hypertension, uncomplicated & $9226(50.6)$ & $1321(53.9)$ & .002 \\
\hline Hypertension, complicated & $900(4.9)$ & $198(8.1)$ & $<.001$ \\
\hline Solid tumor without metastasis & $15,343(84.1)$ & $2189(89.4)$ & $<.001$ \\
\hline
\end{tabular}

POSVT, Postoperative supraventricular tachycardia; $Q$, quartile; $C I$, confidence interval.

and means, medians, and interquartile ranges for continuous variables. The differences in preoperative and postoperative characteristics between patients with versus without POSVT were evaluated using a 2-sample $t$ test, the Pearson $\chi^{2}$ statistic, and Kruskal-Wallis analysis of variance, as appropriate.

Logistic regression was used to identify factors associated with newonset POSVT. Factors of interest were identified a priori; these included baseline patient demographics, state, comorbidities from the Elixhauser Comorbidity Index, and postoperative pulmonary complications. Variables with $P \leq .05$ in bivariate analyses were kept in the multivariable logistic regression model. To analyze the association of POSVT with outcomes, we constructed multivariable logistic regression models for postoperative stroke, in-hospital mortality, and 30-day readmission, and a multivariable linear regression model for log-transformed LOS.

Models were adjusted for statistically significant variables that had a bivariate association with each outcome $(P<.05)$, from among patient demographics, chronic comorbidities, procedure type (open lobotomy and minimally invasive lobectomy), and postoperative pulmonary complications. Generalized estimating equations with exchangeable variance estimation were used to account for the clustering of outcome measures at the hospital level. Adjusted odds ratios (ORs) and 95\% confidence intervals (CIs) of POSVT were calculated. SAS version 9.3 (SAS Institute Inc, Cary, NC) was used to perform all statistical analyses. All $P$ values are 2-sided.

\section{RESULTS}

From the time period studied (2009-2011), we identified a total of 23,043 pulmonary lobectomies, of which 20,695 records met the study criteria (SVT not present on admission, nonmissing age and gender, and age $\geq 18$ years). A relatively equal distribution was found among California $(\mathrm{n}=7226[34.9 \%])$; Florida $(\mathrm{n}=7019[33.9 \%])$; and New York $(\mathrm{n}=6450[31.2 \%])$. Most were performed as open lobectomies $(\mathrm{n}=12,154[58.7 \%]) ;(\mathrm{n}=8541$ [41.3\%]) were minimally invasive lobectomies, including $7611(36.8 \%)$ performed using video-assisted thoracoscopic surgery, and 930 (4.5\%) using robot-assisted thoracoscopic surgery.

Most patients ( $\mathrm{n}=11,335$ [54.8\%]) were women; and white $(\mathrm{n}=15,921[76.9 \%])$. The median age for the cohort was 67 (interquartile range: 59-74) years. The median modified Charlson/Deyo index of this cohort was 3 (interquartile range: 2-4) years. Among comorbidities, 17,617 patients $(85.1 \%)$ had a diagnosis of cancer, 8965 patients $(43.3 \%)$ were noted to have chronic obstructive pulmonary disease, and 479 patients $(2.3 \%)$ had congestive heart failure.

The overall complications rate was $39.7 \%$ in the open lobectomy group, and $31.5 \%$ in the minimally invasive lobectomy group; these were pulmonary complications in $36.5 \%$ and $29.3 \%$ of patients in these groups, respectively. Among these 20,695 patients undergoing pulmonary lobectomy, 2449 patients $(11.8 \%)$ were documented as having newonset POSVT. This total included 1116 (5.4\%) patients who had POSVT as an isolated complication (excluding stroke), and 1333 (6.4\%) patients who had POSVT with other complications. We compared patients with $(\mathrm{n}=2449)$ versus without $(\mathrm{n}=18,246)$ POSVT, to determine differences in baseline characteristics between the 2 cohorts. Table 1 displays the characteristics of patients undergoing pulmonary lobectomy, stratified by POSVT status.

We next performed multivariable analysis to determine independent factors associated with new-onset POSVT after pulmonary lobectomy (Table 2). These included: demographic distinctions (increasing age, male gender, white race); comorbidities (higher Deyo index, hypertension, chronic obstructive pulmonary disease, congestive heart failure, valvular heart disease); and perioperative factors (open vs minimally invasive lobectomy, pulmonary complications).

We further sought to examine whether POSVT was associated with outcome variables, in particular, in-hospital stroke, in-hospital mortality, LOS, and readmission. We performed a multivariable analysis for each outcome, adjusting for age, gender, race, comorbidities, and acute perioperative factors (Figure 1). An independent association was found of POSVT with increased risk for stroke (OR 1.74; 95\% CI: 1.03-2.94); in-hospital mortality (OR 
TABLE 2. Factors associated with postoperative supraventricular tachycardia among pulmonary lobectomy patients

\begin{tabular}{lcc}
\hline \multicolumn{1}{c}{ Variable } & OR $(\mathbf{9 5} \%$ CI $)$ & $\boldsymbol{P}$ value \\
\hline Patient demographics & & \\
$\quad$ Male gender & $1.44(1.31,1.59)$ & $<.001$ \\
Age (y) & Referent & $<.001$ \\
$18-54$ & $2.34(1.84,2.99)$ & $<.001$ \\
$55-64$ & $4.18(3.11,5.62)$ & $<.001$ \\
$65-74$ & $6.21(4.56,8.46)$ & $<.001$ \\
$\geq 75$ & & \\
Race & Referent & $<.001$ \\
White & $0.61(0.50,0.74)$ & $<.001$ \\
Black & $0.59(0.49,0.71)$ & $<.001$ \\
Hispanic & $0.80(0.66,0.96)$ & .020 \\
Other & & \\
Comorbidities & $1.31(1.04,1.64)$ & .020 \\
Congestive heart failure & $1.79(1.43,2.24)$ & $<.001$ \\
Valvular disease & $2.58(1.77,3.78)$ & $<.001$ \\
Pulmonary circulation disorders & $1.17(1.07,1.26)$ & $<.001$ \\
Chronic obstructive pulmonary & & \\
$\quad$ disease & & $<.001$ \\
Perioperative & $1.21(1.09,1.34)$ & \\
Surgical approach: thoracotomy vs & & \\
$\quad$ minimally invasive & $1.95(1.75,2.18)$ & $<.001$ \\
Pulmonary complications & & \\
\hline Data are from multivariable logistic regression analysis, excluding 274 & patients \\
(1.32\%) with missing race variable. OR, Odds ratio; $C I$, confidence interval. &
\end{tabular}

1.85; 95\% CI: $1.45-2.35$ ); LOS (OR 1.33; 95\% CI: 1.29 1.37 ); and 30-day readmission (OR 1.29 ; $95 \% \mathrm{CI}$ : $1.04-$ $1.60)$.

We sought to further examine whether the context of POSVT in regard to other complications was important, particularly whether isolated POSVT is associated with adverse outcomes. The patient cohort was divided into 4 groups: (1) no POSVT or other complications; (2) only POSVT as a complication; (3) those with other complications but no POSVT; and (4) other complications in addition to POSVT (Table 3).

For the purpose of the study, any complication, as determined from ICD-9 codes, was included. From the SIDHCUP database, the temporal sequence of complications cannot be identified. Patients without any other complications, except for stroke, had a lower overall rate of POSVT $(\mathrm{n}=13,180[8.5 \%])$ than did patients with other complications $(\mathrm{n}=7515[17.7 \%] ; P<.0001)$. A comparison of patients who had no complications, with those who did have isolated POSVT but no other complications, showed no difference for in-hospital mortality. Patients with isolated POSVT had significantly higher rates of postoperative stroke, longer LOS (6 vs 5 days), and higher rates of 90day readmission $(20.3 \%$ vs $16.6 \%)$.

However, isolated POSVT patients were older (median age: 72 vs 67 years; $P<.001$ ) and had more cumulative comorbidities (mean Charlson/Deyo index: 3.69 vs 3.42;

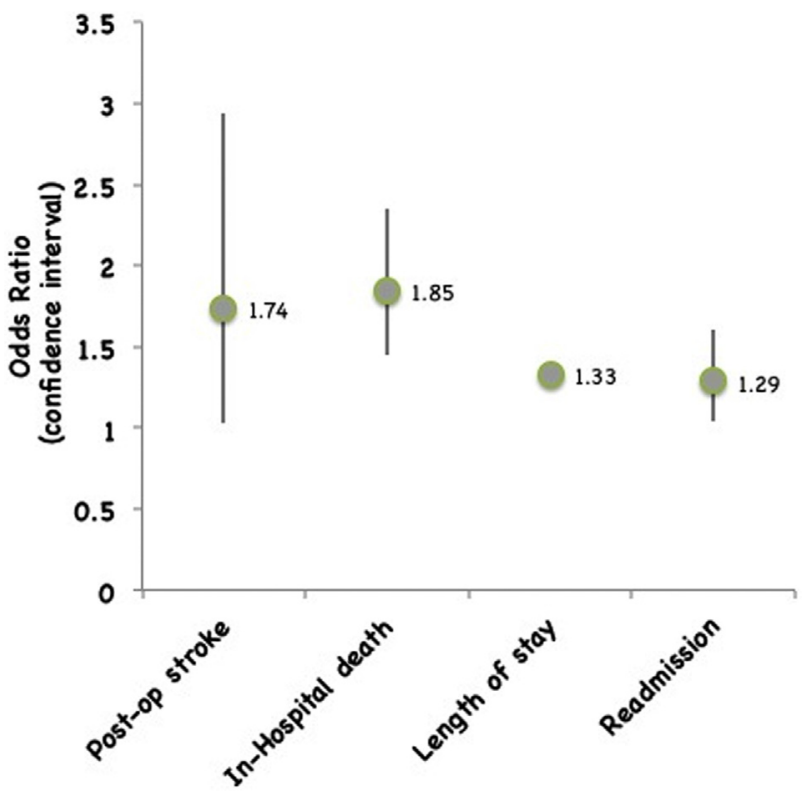

FIGURE 1. Association between postoperative supraventricular tachycardia and adverse outcomes after pulmonary lobectomy. Odds ratios ( $\mathrm{cir}$ cles) and confidence intervals (bars) are presented for postoperative stroke $(P=.04)$, in-hospital death $(P<.001)$, length of stay $(P<.001)$, and 30 -day readmission $(P=.02)$. Post-op, Postoperative.

$P=.001)$. We therefore performed distinct multivariable analyses in this cohort, to determine whether POSVT had independent associations with stroke, mortality, or LOS. However, the number of events $(\mathrm{n}=28)$ was not sufficient to determine an association with stroke. An independent association was found, of POSVT with prolonged LOS (OR 1.33 ; $95 \%$ CI: $1.29-1.37)$ and with 30-day readmission (OR 1.29; 95\% CI: 1.04-1.60).

Patients with POSVT who had other complications did quite poorly, even compared with other patients who had complications but did not have POSVT. The POSVT patients had: higher rates of stroke $(1.5 \%$ vs $0.8 \%$, $P=.009)$; higher in-hospital mortality $(7.7 \%$ vs $3.7 \%$, $P<.001)$; longer median LOS (10 vs 7 days, $P<.001$ ); higher rates of 90 -day readmission $(27.2 \%$ vs $22.2 \%$, $P<.001)$; and higher rates of readmission with stroke $(1.8 \%$ vs $0.8 \%, P=.001)$.

\section{DISCUSSION}

Postoperative SVT has long been recognized as a common complication after thoracic surgical procedures and has been found to be associated with increased morbidity, mortality, LOS, and cost, in single-institutional studies ${ }^{1,6}$ and in a study by Onaitis and colleagues ${ }^{3}$ using the STS General Thoracic Surgery Database. We are not aware of previous studies using insurance databases to validate prospectively reported rates of POSVT and associated complications after noncardiac thoracic surgery. However, the 
TABLE 3. Clinical impact of isolated versus complicated postoperative supraventricular tachycardia

\begin{tabular}{lcccrrr}
\hline \multicolumn{1}{c}{ Outcome } & $\begin{array}{c}\text { No SVT or other } \\
(\mathbf{n}=\mathbf{1 2 , 0 6 4 )}\end{array}$ & $\begin{array}{c}\text { Isolated SVT, no other } \\
(\mathbf{n = 1 1 1 6})\end{array}$ & $\boldsymbol{P}$ value & $\begin{array}{c}\text { No SVT, with other } \\
(\mathbf{n}=\mathbf{6 1 8 2})\end{array}$ & $\begin{array}{c}\text { SVT with other } \\
(\mathbf{n}=\mathbf{1 3 3 3})\end{array}$ & $\boldsymbol{P}$ value \\
\hline Postoperative stroke & $22(0.2)$ & $<11(<1.0)^{*}$ & $<.05^{*}$ & $47(0.8)$ & $20(1.5)$ \\
In-hospital mortality & $26(0.2)$ & $<11(<1.0)^{*}$ & $>.70^{*}$ & $229(3.7)$ & .009 \\
Median length of stay (Q1; Q3) & $5(4 ; 6)$ & $6(5 ; 8)$ & $<.001$ & $7(5 ; 12)$ & $103(7.7)$ & $<.001$ \\
90-d readmission & $1807(16.6)$ & $205(20.3)$ & .003 & $1207(22.2)$ & $309(27.2)$ & $<.001$ \\
90-d readmission with stroke & $69(0.6)$ & $14(1.4)$ & .006 & $41(0.8)$ & $20(1.8)$ & .001 \\
\hline Vin $)$
\end{tabular}

Values are n (\%), unless otherwise indicated. "Other" indicates other complications. SVT, Supraventricular tachycardia; $Q$, quartile. *The Healthcare Cost and Utilization Project Data Use Agreement prohibits the reporting of fewer than 11 observations. Sample size and $P$ values are masked to prevent identification.

SID-HCUP database has been used to determine rates of new-onset atrial fibrillation in patients hospitalized for severe sepsis, and to determine associated morbidity and mortality. ${ }^{13}$ In that study, patients with new-onset atrial fibrillation were at increased risk for stroke and death.

In the current study, we sought to use the SID-HCUP database to confirm self-reported incidences of POSVT in patients undergoing pulmonary lobectomy, ${ }^{1-3}$ define demographic characteristics that are associated with higher rates of POSVT, and determine the association of POSVT with adverse outcomes, including stroke, in-hospital mortality, LOS, and readmission. We acknowledge that limitations of the data include lack of temporal descriptors, and absence of oncologic details and clinical details, such as pulmonary function, performance status, and medication use.

Although such limitations are inherent in using administrative databases, previous studies have demonstrated that reported rates of complications using claims data can approximate those reported in prospective databases. ${ }^{14,15}$ Enomoto and colleagues ${ }^{14}$ suggested that clinical and administrative databases may be discordant; however, their rates of reported cardiovascular complications were within $2 \%$ of each other. Campbell and colleagues ${ }^{15}$ reported that ICD-9-based reporting was actually more accurate than prospective data accrual for capturing new cardiac events. Further, although claims data may be less sensitive than prospective thoracic surgical databases for capturing episodes of new-onset POSVT, this decreased sensitivity may be an advantage if only medically significant episodes of POSVT are recorded by billing specialists.

Patients with clinically insignificant, untreated, short runs of atrial tachycardia, and those not captured by telemetry, would likely not have been coded as having POSVT. The rate at which such prospective, self-reported, surgical databases include these patients is unclear. In our study, the reported overall rate of complications was slightly higher than that reported from the STS database, ${ }^{16}$ and slightly lower than that reported from the Nationwide Inpatient Sample database. ${ }^{17}$

To attempt to homogenize our patient cohort from the SID-HCUP dataset in which cancer staging details are not available, we elected to focus only on patients undergoing pulmonary lobectomy, rather than including other lung resections, such as wedge resection, segmentectomy, or pneumonectomy. A well established finding is that the more extensive the lung resection, the higher the risk of POSVT. ${ }^{1,3}$ For example, in the study by Onaitis and colleagues, ${ }^{3}$ compared with lobectomy, pneumonectomy and bilobectomy had ORs of 2.01 and 1.64, respectively, in the predictive model used.

The current study's focus on lobectomy alone should allow patient characteristics, rather than operation performed, to dominate association models. We found that patient characteristics associated with POSVT include age, male gender, white race, and history of valvular heart disease, congestive heart disease, pulmonary circulation disorders, and chronic obstructive pulmonary disease. The demographic associations with POSVT were all noted in the STS study, and in previous single-institution studies. ${ }^{1-3,5}$ The effect of congestive heart failure and of chronic obstructive pulmonary disease were not apparent in the STS study, although evaluation of both of these variables suffered from the absence of certain data points in the STS database. ${ }^{3}$ We believe that these 2 comorbidities are likely to increase the risk of POSVT.

The surgical approach chosen, open lobectomy versus minimally invasive lobectomy (either video- or roboticassisted thoracoscopic surgery), seemed to affect the rate of POSVT as well (OR 1.18). The association of thoracotomy with increased POSVT has been demonstrated in propensity-matched studies from the $\mathrm{STS}^{16}$ and the Nationwide Inpatient Sample database, ${ }^{17}$ although the surgical approach was not significant in the STS study by Onaitis and colleagues. ${ }^{3}$ Given this contemporary study, which included a higher rate of minimally invasive surgery than previous studies, an effect seems likely.

These data make abundantly clear that the risk of POSVT in patients undergoing pulmonary lobectomy is variable. Although in the recently published guidelines from the AATS, all patients undergoing lobectomy were deemed to be at high risk $(>15 \%)$ for postoperative atrial arrhythmias, we suggest that risk is best characterized by a spectrum that should be evaluated before considering monitoring and prevention strategies for individual patients. ${ }^{7}$ We support development of a risk calculator, for general use on the STS website, as suggested by Onaitis and colleagues. ${ }^{3}$ 
Such a risk calculator could be used to stratify patients into groups, for prospective studies evaluating the intensity of monitoring of postoperative patients, and for studies evaluating prophylaxis against POSVT.

As noted in previous studies, the consequences of POSVT seem to be significant. As patients who develop POSVT are older and have more comorbidities, direct causal effects of POSVT on adverse outcomes are sometimes difficult to identify. We have no data on the timing or nature of stroke or other complications with regard to POSVT. However, in our multivariable model, adjusted for numerous potential confounding factors, POSVT was independently associated with stroke, in-hospital mortality, LOS, and readmission.

This finding confirms those of previous studies. ${ }^{1-3}$ In particular, the consequences of POSVT when it occurs with other complications seem to be particularly dire, with a stroke rate of $1.5 \%$, a median LOS of 10 days, and an in-hospital mortality rate of $7.7 \%$. From the coding in the SID-HCUP database, determining whether POSVT preceded or followed other complications is not possible. Nonetheless, when patients develop POSVT and other complications, managing them in a carefully monitored setting seems appropriate, as well as giving consideration to anticoagulation, when safe to do so.

In contrast to those high-risk patients who have multiple complications, the impact of "uncomplicated" POSVT is less clear. Atrial arrhythmias have been suggested to be transient and relatively harmless in this setting. ${ }^{3}$ We sought to evaluate this possibility by defining a group of patients who have uncomplicated POSVT.

We excluded stroke as a complication, to determine its association with POSVT. We selected patients who were documented as having no other postoperative complications other than atrial fibrillation, atrial flutter, or paroxysmal atrial tachycardia. Among our cohort, $5.4 \%$ of patients undergoing pulmonary lobectomy had uncomplicated POSVT. In this setting, POSVT did not increase inhospital mortality. However, POSVT was still associated with an increase in the incidence of stroke, median LOS, and readmission. This finding suggests that even POSVT without complications makes care of these pulmonary lobectomy patients more complex and puts them at risk for adverse outcomes.

\section{Limitations}

We are left with the question of whether guidelines and evidence-based medicine can be used to reduce the rate of POSVT and associated adverse outcomes in patients undergoing pulmonary lobectomy. Unfortunately, the SID-HCUP database does not contain any information pertaining to medical prophylaxis or to management algorithms for POSVT, which is certainly a limitation of the study. Other limitations are lack of cancer staging information, lack of detailed comorbidity information, and unmeasured variability in postoperative monitoring for POSVT.

Additionally, ICD-9 codes lack detailed, standard clinical definitions that can be universally applied, and are thus open to coding interpretation. Nevertheless, we believe that such ambiguity of definitions is apparent in prospective databases with regard to POSVT. The administrative data used here, carefully evaluated to exclude preoperative diagnoses of SVT, are widely generalizable and not restricted to the outcomes of specialized, experienced centers. The overall rate of POSVT was remarkably similar to that reported for patients in the STS database study $(11.8 \%$ vs $12.6 \%)$, in which $87.5 \%$ of patients underwent lobectomy.

\section{CONCLUSIONS}

Based on our findings, we believe that further studies are needed to evaluate the role of rate control, rhythm conversion, and anticoagulation in these patients, ideally in patients segregated into low- and high-risk cohorts. Among high-risk patients, POSVT may not be as preventable as we like to think, given its dependence on nonmodifiable demographic variables and clinical characteristics.

\section{Conflict of Interest Statement}

A.U.B., R.Z., and P.M.F. have a financial interest in the company Analytical Care. All other authors have nothing to disclose with regard to commercial support.

You can watch a Webcast of this AATS meeting presentation by going to: http://webcast.aats.org/2015/Video/ Tuesday/04-28-15_4E_1100_Stiles.mp4.

\section{References}

1. Vaporciyan AA, Correa AM, Rice DC, Roth JA, Smythe WR, Swisher SG, et al Risk factors associated with atrial fibrillation after noncardiac thoracic surgery: analysis of 2588 patients. J Thorac Cardiovasc Surg. 2004;127:779-86.

2. Passman RS, Gingold DS, Amar D, Lloyd-Jones D, Bennett CL, Zhang H, et al. Prediction rule for atrial fibrillation after major noncardiac thoracic surgery. Ann Thorac Surg. 2005;79:1698-703.

3. Onaitis M, D'Amico T, Zhao Y, O'Brien S, Harpole D. Risk factors for atria fibrillation after lung cancer surgery: analysis of the Society of Thoracic Surgeons general thoracic surgery database. Ann Thorac Surg. 2010;90:368-74.

4. Ciszewski P, Tyczka J, Nadolski J, Roszak M, Dyszkiewicz W. Lower preoperative fluctuation of heart rate variability is an independent risk factor for postoperative atrial fibrillation in patients undergoing major pulmonary resection. Interact Cardiovasc Thorac Surg. 2013;17:680-6.

5. Ivanovic J, Maziak DE, Ramzan S, McGuire AL, Villeneuve PJ, Gilbert S, et al. Incidence, severity and perioperative risk factors for atrial fibrillation following pulmonary resection. Interact Cardiovasc Thorac Surg. 2014;18:340-6.

6. LaPar DJ, Speir AM, Crosby IK, Fonner E Jr, Brown M, Rich JB, et al. Postoperative atrial fibrillation significantly increases mortality, hospital readmission, and hospital costs. Ann Thorac Surg. 2014;98:527-33.

7. Frendl G, Sodickson AC, Chung MK, Waldo AL, Gersh BJ, Tisdale JE, et al 2014 AATS guidelines for the prevention and management of perioperative atrial fibrillation and flutter for thoracic surgical procedures. J Thorac Cardiovasc Surg. 2014;148:e153-93.

8. Fernando HC, Jaklitsch MT, Walsh GL, Tisdale JE, Bridges CD, Mitchell JD, et al. The Society of Thoracic Surgeons practice guideline on the prophylaxis and management of atrial fibrillation associated with general thoracic surgery: executive summary. Ann Thorac Surg. 2011;92:1144-52. 
9. Agency for Healthcare Research and Quality. Healthcare Cost and Utilization Project. Available at: http://www.hcup-us.ahrq.gov/sidoverview.jsp. Accessed date January 5, 2016.

10. Stiles BM, Poon A, Giambrone GP, Gaber-Baylis LK, Wu X, Lee PC, et al. Incidence and factors associated with hospital readmission following pulmonary lobectomy. Ann Thorac Surg. December 22, 2015 [Epub ahead of print].

11. Deyo RA, Cherkin DC, Ciol MA. Adapting a clinical comorbidity index for use with ICD-9-CM administrative databases. J Clin Epidemiol. 1992;45:613-9.

12. Quan H, Li B, Couris CM, Fushimi K, Graham P, Hider P, et al. Updating and validating the Charlson Comorbidity Index and score for risk adjustment in hospital discharge abstracts using data from 6 countries. Am J Epidemiol. 2011;173: 676-82.

13. Walkey AJ, Wiener RS, Ghobrial JM, Curtis LH, Benjamin EJ. Incident stroke and mortality associated with new-onset atrial fibrillation in patients hospitalized with severe sepsis. JAMA. 2011;306:2248-54.

14. Enomoto LM, Hollenbeak CS, Bhayani NH, Dillon PW, Gusani NJ. Measuring surgical quality: a national clinical registry versus administrative claims data. $J$ Gastrointest Surg. 2014;18:1416-22.

15. Campbell PG, Malone J, Yadla S, Chitale R, Nasser R, Maltenfort MG, et al. Comparison of ICD-9-based, retrospective, and prospective assessments of perioperative complications: assessment of accuracy in reporting. J Neurosurg Spine. 2011;14:16-22.

16. Paul S, Altorki NK, Sheng S, Lee PC, Harpole DH, Onaitis MW, et al. Thoracoscopic lobectomy is associated with lower morbidity than open lobectomy: a propensity-matched analysis from the STS database. J Thorac Cardiovasc Surg. 2010;139:366-78

17. Paul S, Sedrakyan A, Chiu YL, Nasar A, Port JL, Lee PC, et al. Outcomes after lobectomy using thoracoscopy vs thoracotomy: a comparative effectiveness analysis utilizing the Nationwide Inpatient Sample database. Eur J Cardiothorac Surg. 2013;43:813-7.

Key Words: pulmonary lobectomy, postoperative supraventricular tachycardia

\section{Discussion}

Dr Ara Vaporciyan (Houston, Tex). Brendon, I just wanted to congratulate you and your colleagues on expanding our understanding of this complication; it is a very common event after thoracic surgery. The findings that you have added really substantiate some of the findings of other investigators, and they have added new information and highlighted some important points that I think we can share here in this meeting.

Obviously, one of the big advantages of using an administrative database is the large sample size (approximately 20,000 patients) and leveraging that to produce a consistent group of patients. By concentrating on the lobectomy patients only, you were able to exclude some confounding factors like pneumonectomy, which have a clear impact on the incidence of atrial fibrillation and may mask some of the more subtle factors that might be identified. Of course, there are obvious limitations with these studies. You have presented them in your paper, and other investigators have also identified those. But accepting these advantages and disadvantages, I think you really did focus on the things that you can support with your data and tried to avoid areas where the data were probably weak.

There are some findings that are really interesting and should be highlighted to the audience. I had the added advantage of being able to see the manuscript. I think one of the most interesting factors is just the rate of minimally invasive surgery. This is a national database, and you were able to demonstrate that now more than $40 \%$ of our patients are getting minimally invasive lobectomies; and just 4 years ago, in a paper by Onaitis, that came out of the Society for Thoracic Surgeons (STS) database, which clearly concentrate data from thoracic surgeons, that rate was just over $20 \%$. In addition, your data were actually drawn from the period 2009 to 2011, which corresponds pretty closely with that in Onaitis's paper. So you were able to demonstrate either a difference between national databases and our STS database or a rapid expansion in the penetration of minimally invasive surgery.

The most important thing you showed is the association with stroke. Because of the large size of the database, and because you were able to focus on lobectomies, you were able to show the association of stroke. We must accept the limitations of not knowing which came first, but still, I think that was an important finding.

I do have a few questions before I relinquish my role as a discussant. The first question has to do with methodology. I totally understand your desire to develop some sort of predictive model to be able to identify who is going to develop atrial fibrillation so we can better guide studies. The part I found confusing was the inclusion of postoperative pulmonary complications in that model. Clearly, postoperative pulmonary complications were predictive of developing atrial fibrillation, but this is a postoperative event, and so I would not want to include it in a model to try to predict who is going to get atrial fibrillation. So as my first question, I would ask you to comment on that decision and whether you would do things any differently.

Dr Stiles. That is a great point, obviously. We talked about this. I think one of the problems with administrative databases like this that are not set up to capture complications, but rather to show costs, is that you have to be very careful about using them as the basis of a predictive model. We were very careful in the article to say these are just associations. We cannot say that this predicts atrial fibrillation.

I think if one were to make a prediction model, which Mark Onaitis and others have suggested on the STS site, then certainly getting rid of pulmonary complications is important. Here we just say that it is associated, and we would not want to use it as a predictive variable. I agree.

Dr Vaporciyan. So the other 2 questions are really an opportunity for you to pontificate, because obviously the data are not going to be in depth, as I said, in an administrative database. The first question is: How do you think your finding of an increased rate of stroke affects your decision to give a patient prophylaxis? Specifically, what type of prophylaxis would you now think is appropriate? There are a number of individuals who have offered amiodarone as a form of prophylaxis, but there are also a number of 
clinicians who may be fearful of using amiodarone, because of its very low but known risk of pulmonary injury, and might avoid its use. Do you think that your data might change that conversation?

Dr Stiles. I hope that these data will have people just think about who they are going to give prophylaxis; I think we can at least agree with that. Again, these data do not really say anything about treatment types or options. I think our group in the past has just given prophylaxis to everybody undergoing lobectomy, which is the suggestion in the AATS guidelines. Clearly, there are gradations of risk, though; some patients probably do not need prophylaxis. Any of those drugs that you choose are fine, I think, and you can debate about each one, but each is also associated with some negative implications-a $10 \%$ to $30 \%$ risk of bradycardia or hypotension, and probably with cost and other issues as well. So I think the key is just choosing who you are going to give prophylaxis. I cannot speak to how you are going to do it.

Dr Vaporciyan. My last question is a chicken-and-egg sort of question. A lot of people have contemplated the fact that atrial fibrillation is an inflammatory complication and therefore is caused by other inflammatory complications like postoperative pneumonia. On the other hand, some believe that atrial fibrillation is a marker for patients who have a higher propensity to develop complications. Based on your readings, and this work, do you have anything to add to that conversation?

Dr Stiles. That is a tough question. There are a couple of papers that evaluated sequence of atrial fibrillation or other arrhythmias after postoperative events. There is a good one from Cleveland that you had pointed out to me-they saw that approximately $60 \%$ to $70 \%$ of the SVT came after the other complications. From our date, we obviously cannot focus in any further on that issue. I think, certainly, inflammation is related. The fact that demographics, such as white race and male gender, are so important tells you that there must be some sort of basis for that.

Dr Vaporciyan. Great. I will leave the discussion open to the audience.

Dr G. Hossein Almassi (Milwaukee, Wis). It is great to see a talk about atrial fibrillation that comes from the general thoracic side, because our group and many others have reported atrial fibrillation problems with cardiac patients, regardless of its duration, and new postoperative atrial fibrillation has always been associated with short- and long-term adverse events and even long-term survival. Congratulations for presenting these data that focus on the general thoracic side. As mentioned already, the newer version of the STS recommendation is more favorable toward amiodarone, versus the 2010 guidelines, which tried to not recommend it.

What you showed is that the stroke rate is high; the rates of all other complications are also high. Is it a question of chicken or egg, in terms of cause? Is the sick patient the reason, or are the other factors responsible for it?; and we do not know the answer.

I understand the concern for having lung resection and starting amiodarone, but most of the results regarding complications with amiodarone were in patients who were on long-term amiodarone. We are talking about a short term, a few weeks, for a patient who develops postoperative atrial fibrillation. So I think there should be more discussion in this regard, to come to a consensus to prevent these adverse events for patients who undergo surgical procedures on the lung, or the esophagus, for that matter.

Dr Stiles. Well, I agree. Thanks for your comments. I am certainly not the first to talk about this. Both the AATS and STS have been actively involved, and several institutions have written good papers about this as well.

Dr Vaporciyan. There have actually been some publications evaluating genetic factors that may play a role in the development of pulmonary and any other inflammatory complications. Our group has actually published some work on single-nucleotide-polymorphism analysis for inflammatory cytokines, such as tumor necrosis factor and interleukin-1, and we did notice that patients who had specific single-nucleotide-polymorphisms had a much higher incidence. Of course, those data have not been validated; they were from a single institution, but there are other similar studies done for other inflammatory diseases that do show that there is a genetic predisposition to developing some inflammatory events.

Dr Joseph S. Coselli (Houston, Tex). Just a quick question: With stroke being the primary morbidity of focus, did your database separate those who had hemorrhagic versus embolic strokes, to try to determine whether or not the problem was the disease or the treatment?

Dr Stiles. It did separate these by the ICD-9 codes, and we excluded the hemorrhagic strokes and evaluated only the embolic strokes. 